\title{
tic\&société
}

Vol. 8, No 1-2 | 1er semestre 2014 et 2ème semestre 2014

Quelles perspectives critiques pour aborder les TIC ?

\section{Les recherches critiques appliquées dans le champ des TICN : chimère ou percée ? Éléments de réflexion}

Jacob T. Matthews et Jérémy Joseph Vachet

\section{(2) OpenEdition \\ Journals}

Édition électronique

URL : http://journals.openedition.org/ticetsociete/1454

DOI : 10.4000/ticetsociete. 1454

\section{Éditeur}

Association ARTIC

\section{Référence électronique}

Jacob T. Matthews et Jérémy Joseph Vachet, « Les recherches critiques appliquées dans le champ des TICN : chimère ou percée ? Éléments de réflexion », tic\&société [En ligne], Vol. 8, № 1-2। 1er semestre 2014 et 2ème semestre 2014, mis en ligne le 02 juin 2014, consulté le 30 avril 2019. URL : http://journals.openedition.org/ticetsociete/1454 ; DOI : 10.4000/ticetsociete.1454 
tic\&société - 8 (1-2), 2014

Les recherches critiques appliquées dans le champ des TICN : chimère ou percée? Éléments de réflexion

Jacob T. MATTHEWS

CEMTI

Université Paris 8,

Maison des Sciences de l'Homme Paris Nord

jacob.matthews@univ-paris8.fr

Jérémy Joseph VACHET

LABSIC

Université Paris 13,

Maison des Sciences de l'Homme Paris Nord

acetteadresse@gmail.com 


\title{
Les recherches critiques appliquées dans le champ des TICN : chimère ou percée? Éléments de réflexion.
}

\begin{abstract}
Jacob T. Matthews est docteur en sciences de l'information et de la communication (2006) et maître de conférences à l'Université Paris 8. II est également membre du CEMTI et de l'observatoire des mutations des industries culturelles (OMIC). Ses travaux s'inscrivent au croisement de la théorie critique et de la socio-économie des industries de la culture et de la communication. II est l'auteur, avec Vincent Rouzé et Jérémy Vachet de La Culture par les foules ? Le crowdfunding et le crowdsourcing en question (2014) et a co-écrit, avec Philippe Bouquillion, Le Web collaboratif : mutations des industries de la culture et de la communication (2010).
\end{abstract}

Jérémy Joseph Vachet est doctorant au LabSIC à l'Université Paris 13, sous la direction de Philippe Bouquillion. Ses recherches doctorales analysent la nature et le statut du travail artistique ainsi que le rôle que la critique artiste joue dans le développement du capitalisme avancé. Ses recherches de terrain ont été menées parmi des réseaux de travailleurs "créatifs" au sein de grande métropoles mondiales. A ce titre, il a été accueilli en tant que visiting scholar à I'Université du Massachusetts à Amhurst (2014) et à l'Université de Californie à Berkeley (2013).

Résumé : En France, les sciences humaines et sociales (SHS), et notamment les travaux sur les technologies d'information et de communication numériques (TICN), sont confrontés au développement d'incitations publiques en faveur de consortia entre entreprises privées et équipes de recherche. Pour des chercheurs se réclamant d'approches critiques et ne souhaitant pas éluder la question de l'application (sociale, socio-technique, voire politique) de leurs travaux, la participation à ce type de partenariat suscite d'importantes interrogations. A cet égard, notre article n'a pas pour but d'exposer des conclusions de travaux; il s'agit avant tout de rendre compte de réflexions que nous ont inspirées plusieurs expériences de recherches menées au sein de consortia. En somme, nous nous demandons si le choix de "s'immerger " au cœur de mutations socio-techniques pour en faire l'analyse critique se justifie, compte tenu des conditions dans lesquelles s'effectue cette recherche.

Nos constats partent de l'évolution de contraintes institutionnelles qui placent le chercheur critique dans une position ambiguë, tiraillé entre le souci de distanciation vis-à-vis de ses objets et la proximité avec ces derniers que 
Les recherches critiques appliquées dans le champ des TICN : chimère ou percée ? Éléments de réflexion

suppose la recherche en consortium. Par-delà cette problématique, nous avons observé une rationalisation du travail de recherche au travers une responsabilisation croissante du chercheur et la généralisation du travail par projet. Par extension, c'est l'injonction au développement de logiques entrepreneuriales et l'insertion plus générale de la recherche au sein du paradigme des « industries créatives » que nous interrogeons.

Mots clés: Recherche critique, recherche appliquée, web collaboratif, consortium, TICN, industries créatives.

Abstract : The humanities and social sciences (especially research relating to information and communication technologies) in France, have seen an expansion in public calls for the creation of consortia between universities and private companies. In this context, the principles and aims of critical research finds itself marginalized. This article is not meant to present the findings of a specific investigation. It seeks, instead, to advance reflections on critical research inspired by experiences within research consortia. We ask whether positioning oneself within sites of socio-technical change is still justified given the conditions in which critical research now takes place. Our findings stem from the evolution of institutional constraints that put critical researchers in an ambiguous position. In addition to tensions between proximity and distance, we have observed the rationalization of research work through the the placing of increased volume of responsibilities on researchers and the generalization of project-based work. Ultimately, our work questions connection between the growing onus placed on developing entrepreneurial mindset and the broader integration of research within the "creative industries" paradigm.

Keywords : critical research, applied research, collaborative web, consortium, ICNT, creative industries.

Resumen : En Francia, en el seno de las ciencias sociales y humanas - y más en especial en los trabajos sobre las tecnologías de información y de comunicación digital (TICD) - hemos asistido estos últimos años a la multiplicación de los incentivos públicos para favorecer las relaciones entre empresas privadas y Universidades, bajo la forma de consortia. En este contexto, se cuestiona el principio de las finalidades de las investigaciones críticas.

Este artículo no tiene como objetivo mostrar resultados de trabajos particulares sino de realizar reflexiones inspiradas por experiencias en los consortia que consideramos. Se plantea la cuestión de si la elección de posicionarse en el centro de las mutaciones socio-técnicas para efectuar el análisis crítico puede 
justificarse, dadas las condiciones en las cuales se efectúa dicha investigación. En suma, la cuestión esencial es si de cara a hacer un análisis crítico se justifica la opción de la «inmersión » en el corazón del cambio socio-técnico, dadas las condiciones en las que tal investigación se lleva a cabo. La conclusión parte de la evolución de las restricciones institucionales que colocan al investigador crítico en una posición ambigua, ya que por una lado, debe mantener una distancia respecto a los objetos a la vez que debe mantener una cercanía a estos últimos, como consecuencia de pertenecer al consortia. Por otro lado, hemos observado una racionalización de los procedimientos de investigación, visible tanto en el incremento de la responsabilidad como en la generación de trabajos basados en proyectos. Finalmente se analiza el desarrollo de lógicas de gestión empresarial y de la generalización del paradigma de "industrias creativas" en la investigación.

Palabras clave: investigación crítica, investigación aplicada, web de colaboración, consortium, TICD, industrias creativas. 
Les recherches critiques appliquées dans le champ des TICN : chimère ou percée ? Éléments de réflexion

\begin{abstract}
La procédure des sciences sociales officielles n'est, à peu de choses près, plus qu'une parodie des entreprises qui maintiennent une telle science à flot et qui n'en ont réellement besoin qu'en tant que publicité.
\end{abstract}

\title{
Introduction
}

Theodor Adorno, Messages in a Bottle

Formulées dans un texte initialement destiné à l'ouvrage Minima Moralia (1951), ces réflexions d'Adorno gardent une pertinence certaine, s'agissant notamment des recherches sur les TICN, où discours promotionnels et " analyses " académiques s'entremêlent plus ou moins insidieusement. Dans ce champ des SHS, aussi bien en Europe qu'en Amérique du Nord, les phénomènes du web 2.0, des médias sociaux ou de l'économie collaborative suscitent un engouement considérable. A l'instar d'Henry Jenkins (2006) et de ses traducteurs francophones, ou de Patrice Flichy (2010), un nouveau courant dominant de recherches en communication semble aujourd'hui se structurer, donnant la priorité au recensement (et à la promotion à peine voilée) de formes de création, d'engagement, de partage et de valorisation supposément inédites et propres à l'ère numérique. Nos recherches s'efforcent au contraire de replacer ces phénomènes au sein de perspectives historiques et socioéconomiques plus larges. A cet égard, nous nous inscrivons pleinement dans la perspective que dessine Olivier Voirol, s'agissant des conditions d'une réactualisation de la théorie critique :

«Tout d'abord, elle implique une approche qui décloisonne l'étude des processus de communication en réinscrivant ces derniers dans l'ensemble des processus sociaux (formation des sujets contemporains, modèle de capitalisme actuel, rapports entre culture et économie, etc.). (...). Ensuite, elle suppose de disposer d'un "référent pratique" offrant un point de repère normatif et préthéorique permettant, d'une part, de concevoir des formes émancipatrices de créations culturelles et d'autre part, de mener une critique systématique de ce qui contribue à les oblitérer, les empêcher ou les détruire. (...) Enfin, il convient de disposer d'une méthodologie permettant une "analyse interne" des produits médiatiques et des biens culturels, capable en même temps de relier cette analyse à des processus "externes". ” (Voirol, 2010, p. 32)

Ces trois dimensions - théorique, éthique et méthodologique - font écho à ce qui nous semble constituer l'une des lacunes majeures des recherches 
dominantes sur les TICN : le manque de réflexion sur leurs propres conditions de production (Bourdieu, 1984, p. 65) ${ }^{1}$. Le contexte idéologique et socioéconomique qui caractérise le déploiement massif des TICN au cours des trente dernières années incite pourtant à examiner en profondeur les relations qui lient acteurs industriels et chercheurs. A contrario, comme nous l'avons signalé dans une étude récente sur le web collaboratif, les universitaires sont désormais invités à adopter la fonction de médiateurs entre usagers et acteurs industriels (Bouquillion, Matthews, 2010).

Ces observations générales renvoient à la fois vers les problèmes du financement et des finalités des recherches en SHS, et donc vers la question des bases matérielles de ces recherches, que l'on trouve sous une forme "épurée » dans la citation introductive de Theodor Adorno ${ }^{2}$. Un de postulats du travail que nous présentons ici est que les recherches critiques ne peuvent faire l'économie d'une réflexion générale sur l'évolution de leurs propres conditions de production, de leurs bases matérielles. L'objectif de notre article est de contribuer à cette réflexion, en nous appuyant sur une analyse à partir de travaux menés dans le cadre de plusieurs programmes de recherche appliquée. II s'agit de ce fait d'une contribution singulière, dont le but est avant tout de nourrir les questionnements et discussions qui animent de nombreux chercheurs se réclamant d'approches critiques et néanmoins impliqués au sein de programmes dont on ne saurait pleinement nier le caractère « administratif ».

Dans quelle mesure ces approches sont-elles diluées dans le cadre de consortia associant acteurs industriels et laboratoires de recherche ? Existe-t-il un risque de neutralisation de la critique du capitalisme des industries de la culture et de la communication, du fait de ces modalités de recherche? Comment s'en trouve affectée la position du chercheur? Ces questions se posent sans doute de manière particulièrement aiguë dans le domaine des TICN, où les chercheurs peuvent être eux-mêmes usagers des dispositifs et acteurs des processus étudiés. De plus, à partir des expériences de recherche que nous analysons ici, une question essentielle se dégage : faut-il être au cœur des mutations socio-techniques pour mieux les critiquer?

On sait qu'une des accusations les plus récurrentes face aux approches critiques s'appuie sur leur caractère prétendument « spéculatif », « éloigné de la

\footnotetext{
1 Nous renvoyons ici à la proposition bien connue de Pierre Bourdieu: "la connaissance des conditions de production du produit fait partie, en toute rigueur, des conditions d'une communication rationnelle du résultat de la science sociale. "

${ }^{2}$ Ce constat s'appuie indubitablement sur l'expérience des recherches " administratives " menées durant la période de l'exil étasunien, dont l'ouvrage de David Jenemann, Adorno in America (2007), offre une analyse passionnante.
} 
Les recherches critiques appliquées dans le champ des TICN : chimère ou percée ? Éléments de réflexion

recherche empirique et de la concrétude de l'enquête sur les pratiques culturelles et médiatiques. » (Voirol, 2010, p. 24). Sans vouloir donner trop de crédit à de telles accusations, il nous semble important de préciser brièvement les contours des travaux sur lesquels s'appuie notre réflexion.

II s'agit, en premier lieu, de recherches menées dans le cadre du consortium CrowdBooks (2009-2011), dont le titre correspond précisément à l'appellation d'un prototype de plateforme de production collaborative d'ouvrages littéraires, développé par la société CrowdCulture ${ }^{3}$. Ce projet a bénéficié d'un financement dans le cadre du "volet numérique " du plan de relance gouvernemental de 2009. L'objectif formel des six chercheurs du laboratoire CEMTI ${ }^{4}$ associés au consortium était de prolonger des recherches socio-économiques entamées depuis plusieurs années, tout en mettant leur expertise à disposition de l'entreprise partenaire, en échange de l'accès à un terrain d'observation et d'expérimentation inédit: celui du développement de la plateforme et des usages générés. Comme nous le verrons, ces objectifs n'ont été que partiellement atteints. L'expérience du consortium CrowdBooks $(C B)$ a surtout servi de catalyseur pour un projet autrement plus ambitieux sur le plan scientifique comme du point de vue de l'implication pratique des chercheurs, mais qui n'aura toutefois pas bénéficié d'un financement équivalent au premier.

Ce second programme (2011-2012), que nous évoquerons de façon plus approfondie, s'est également appuyé sur un consortium intitulé Le live collaboratif $(L L C)$, regroupant quatre entités : le CEMTI (représenté par quatre chercheurs); Collablive, structure responsable du développement d'une plateforme web; une association détentrice d'une licence de production de spectacles ; et un studio de conception informatique et infographique. Dans la demande de labellisation du projet scientifique par la Maison des Sciences de l'Homme Paris Nord, nous soulignions qu'il avait pour objectif de contribuer à une recherche appliquée d'un type nouveau, en accompagnant la mise en place d'un dispositif de médiation numérique, tout en prolongeant des recherches critiques ayant permis d'identifier certains risques inhérents à la structuration socio-économique des industries de la culture et de la communication, en termes d'appropriation et de distribution des ressources, de qualité et de diversité des contenus, ou encore du point de vue du renforcement de représentations idéologiques dominantes.

\footnotetext{
${ }^{3}$ Les noms de l'entreprise et du consortium ont été modifiés.

4 Centre d'études sur les médias, les technologies et l'internationalisation (http://www2.univparis8.fr/cemti/).
} 
Les objets de recherche élaborés dans le cadre de ces programmes étaient donc riches du point de vue des approches critiques qu'ils permettaient de mobiliser, tout en fournissant la perspective d'enquêtes empiriques conséquentes. Ils prolongeaient en outre trois thèmes de recherche complémentaires que nous avions déjà largement abordés: l'émergence du web collaboratif, les mutations des industries culturelles, et les évolutions des politiques culturelles. Cet article n'a pas pour objectif d'exposer nos résultats ${ }^{5}$, mais de livrer des réflexions à partir de nos expériences pratiques de chercheurs. A cette fin, nous présentons tout d'abord quelques éléments du contexte général de ces travaux et leurs objectifs fondamentaux, en explicitant notre visée de "mise en application » d'approches critiques. Dans un second temps, tout en avançant certaines pistes d'ouverture, nous aborderons les enjeux et les antagonismes spécifiques à ces expériences de recherche. Ceuxci transparaissent à deux niveaux complémentaires : celui du cadre institutionnel dans lequel ces travaux ont été réalisés, et celui de la position et du rôle du chercheur critique dans le champ des TICN. La conclusion de notre travail s'efforcera de tracer les contours d'une dialectique de la participation et de la distanciation propre aux recherches critiques appliquées.

\section{Des recherches critiques appliquées portant sur le crowdfunding et le crowdsourcing culturels: pourquoi et comment?}

\subsection{Le contexte général de nos travaux sur les plateformes collaboratives}

Les recherches sur lesquelles s'appuie cette réflexion ont porté sur l'émergence de plateformes web dédiées au financement et à la production collaboratifs (crowdfunding et crowdsourcing) dans le domaine des industries de la culture et de la communication. Ces notions se rapportent à la prise en charge collective, par une « foule » d'internautes ponctuellement et virtuellement réunis autour de sites spécialisés, d'activités diverses (financement, conception, édition, production, promotion, distribution) relevant classiquement des acteurs industriels. Le mode de valorisation de ces sites repose sur l'intermédiation, c'est-à-dire sur des commissions perçues principalement à partir des échanges entre porteurs de projets ou commanditaires de travaux, d'un côté, et internautes travailleurs ou financeurs, de l'autre. L'objectif de ces plateformes

\footnotetext{
${ }^{5}$ A cette fin, nous renvoyons le lecteur à l'ouvrage issu de ces travaux, La culture par les foules : le crowdfunding et le crowdsourcing en question (Matthews, Rouzé, Vachet, 2014).
} 
Les recherches critiques appliquées dans le champ des TICN : chimère ou percée ? Éléments de réflexion

est d'administrer et d'optimiser ces formes de travail et de financement, de façon à remplacer (partiellement ou intégralement) l'apport logistique ou financier traditionnellement pris en charge par des producteurs industriels. Ce transfert de charges vers la "foule " permet aux plateformes (et à leurs partenaires industriels) de diminuer le risque lié au caractère incertain de la valeur d'usage des produits culturels, stratégie d'autant plus profitable que les usagers de plateformes participent aux tâches promotionnelles.

Ces recherches ont été conduites dans la continuité de notre étude sur le web collaboratif (Bouquillion, Matthews, 2010) qui analyse ce phénomène à partir de deux angles complémentaires. Premièrement, nous illustrons comment celui-ci tend à renforcer les liens entre industries de la culture et de la communication, d'un côté, et industries des biens et services de consommation, de l'autre. Deuxièmement, le web collaboratif apparaît comme un champ d'expérimentation, permettant aux acteurs industriels d'alimenter, de capter et de mettre en valeur les échanges et les contributions des usagers, justifiant ainsi la viabilité d'un nouveau modèle sociétal, basé sur le marché-réseau. Par conséquent, nous avons émis l'hypothèse que les dispositifs du web collaboratif (tels que les plateformes de crowdfunding et de crowdsourcing culturels) participent simultanément de mutations industrielles structurelles et de processus de production idéologique.

Les enquêtes ${ }^{6}$ menées dans le cadre des programmes CrowdBooks ( $\left.C B\right)$ et Le live collaboratif ( $L L C$ ) montrent comment ces plateformes participent à une intensification des logiques industrielles (financiarisation, marchandisation et rationalisation accrues, responsabilisation individuelle et désengagement de l'État ${ }^{7}$, tout en véhiculant un discours d'affranchissement (empowerment) des internautes, de défense des marges culturelles et de rupture avec l'industrie.

Dans un premier temps ces enquêtes ont visé des plateformes de financement et de production collaboratifs dans l'ensemble des industries culturelles (et même au-delà). Elles se sont ensuite concentrées plus

\footnotetext{
${ }^{6}$ Sur le plan méthodologique, en complément de l'analyse de données secondaires, nous avons privilégié l'entretien semi-directif (avec des échantillons d'usagers et des responsables de plateformes) et l'observation directe (dans le cadre de réunions internes, de présentations publiques et de conférences professionnelles).

${ }^{7}$ Ces points sont directement en lien avec la thèse de Jérémy Joseph Vachet, entamée en 2010 et intitulée Mutations de l'industrie musicale: du D.I.Y. (do it yourself) au D.I.W.O. (do it with others) (co-dirigée par Philippe Bouquillion et Jacob Matthews). Ces recherches doctorales portent sur le phénomène D.I.Y. dans les milieux créatifs, à l'intersection entre discours libertaire et managérial, et par extension sur la manière dont le capitalisme, via les industries culturelles et créatives, reconstruit un travailleur plus adapté à ses besoins présents.
} 
spécifiquement sur la filière de la musique live, en France et en Grande Bretagne, du fait du projet d'accompagnement de la plateforme Collablive, mais également à cause de l'intérêt que nous portons aux évolutions des politiques publiques, dans le cadre de la montée en puissance du discours des "industries créatives" (Bouquillion, 2012). A ce titre nous nous sommes intéressés aux évolutions des politiques publiques vis-à-vis de la filière du spectacle vivant et à l'émergence, en France, de dispositifs dits de "subventionnement de la demande ". Des cartes et chèques culture, émis par des institutions publiques ou des collectivités locales, permettent ainsi à des spectateurs de payer tout ou partie d'un billet de concert dans des salles partenaires. Notre choix de nous intéresser à cette filière fait écho, enfin, à nos travaux antérieurs sur les représentations idéologiques dans les "musiques actuelles », dont une partie significative des enquêtes de terrain avaient été menées dans le milieu de la musique live (Matthews, 2009).

Les recherches réalisées dans le cadre du programme $L L C$ ont illustré comment ces plateformes contribuent à la réorganisation d'une filière jusque là peu structurée industriellement. S'agissant de la musique live, de nombreuses études ont mis en avant la permanence d'un " écosystème " diversifié, marqué par un nombre significatif d'acteurs de taille limitée, fonctionnant souvent sur des territoires restreints et avec des procédures hétérogènes et assez peu codifiées (Guibert, 2006 ; Frith, 2007 ; Brennan \& Webster, 2011 ; Dufy, 2012). A contrario, les tentatives d'implantation de plateformes de financement et de production participatifs s'efforcent d'imposer des pratiques standardisées et plus homogènes en amont (organisation du travail, négociations entre artistes, salles, tourneurs et producteurs) et en aval (captation et gestion de données sur les publics et l'offre de spectacles, prévente de places de concerts et gestion de billetterie, etc). Ces évolutions sont à replacer dans le contexte plus général d'une concentration des acteurs de la filière du spectacle vivant, qui favorise en soi un renforcement de l'industrialisation structurelle; les dispositifs de crowdfunding et de crowdsourcing y jouent d'ores et déjà un rôle significatif.

\subsection{Les objectifs contrastés des consortia CB et LLC}

Nous avons présenté le contexte et les orientations générales des recherches menées dans le cadre des programmes $C B$ et $L L C$. Toutefois, ces travaux se sont tous deux inscrits dans le cadre de partenariats dont il convient de fournir quelques éléments descriptifs. 
Les recherches critiques appliquées dans le champ des TICN : chimère ou percée ? Éléments de réflexion

Dans le premier cas, le CEMTI, représenté par six chercheurs ${ }^{8}$, s'est associé à la société CrowdCulture $(C C)$ dans le cadre d'un appel à projets du "volet numérique » du Plan national de relance de l'économie de 2009. Notre projet visait à concevoir et à tester un prototype de plateforme de financement participatif dédié à l'édition d'ouvrages de fiction, CrowdBooks, conçu sur le modèle du site éponyme de l'entreprise partenaire, qui entrait alors dans sa troisième année d'existence et qui s'était bâtie une réputation déjà solide dans le crowdfunding phonographique. Chaque partenaire reçut une dotation financière distribuée via la Direction générale de la compétitivité, de l'industrie et des services (DGCIS), sur la base d'une répartition préalable des tâches de recherche et de développement. Chacun était donc libre d'organiser ses dépenses sans ingérence de part et d'autre et avec pour seul interlocuteur logistique et financier la DGCIS. Le cahier des charges dont nous avions convenu avec $C C$ permit à l'équipe du CEMTI de fixer ses propres orientations de recherche (dont nous avons exposé certains points saillants ci-dessus). Hormis la préparation de rencontres régulières et l'assemblage de rapports à l'intention de la DGCIS, ce consortium impliqua donc assez peu de contraintes en termes de "rendus " scientifiques. Bien que notre réponse à l'appel d'offre ait été clairement placée sous le signe de la recherche et développement (R\&D), il est incontestable que nous avons bénéficié, au sein de ce consortium, de conditions atypiques ${ }^{9}$. Outre le paiement de missions de recherche, il permit de contribuer au financement de deux thèses, en recrutant temporairement un premier doctorant, puis en rétribuant le travail d'un second (par des modalités que nous exposons plus bas).

Comme nous l'avons souligné en introduction, le second projet de consortium réunissait formellement quatre partenaires dans le but de développer et de lancer une plateforme de financement et de programmation collaboratifs de concerts, Collablive, tout en fournissant à chaque étape de cette

\footnotetext{
8 Ont fait partie de cette équipe dirigée par Jacob Matthews, les enseignants chercheurs titulaires Sylvie Bosser, Françoise Paquienséguy et Vincent Rouzé, ainsi que les doctorants Efstathios Sinos (2009-2011) puis Jérémy Joseph Vachet (2011). L'équipe a en outre été assisté ponctuellement par deux étudiants de Master, Bastien Louessard et Peter Nutsuego, recrutés en tant qu'agents nontitulaires.

${ }^{9}$ Ceci s'explique en grande partie par le fait que la société partenaire avait déjà mis au point son prototype de service (et noué un accord commercial avec un acteur de la filière de l'édition) au moment où nous étions en théorie censés commencer ce travail commun de recherche et développement. Ainsi, contrairement aux partenariats laboratoire/société privée tels qu'ils sont généralement conçus, notre travail ne présentait pas d'intérêt stratégique pour l'entreprise. La mise au point formelle d'un consortium leur permettait avant toute chose de bénéficier d'un financement public significatif.
} 
progression, un terrain de recherche novateur. Porté depuis plusieurs années par un ancien étudiant de l'Université Paris 8 , le projet de plateforme avait peu à peu mûri et comportait des innovations à trois niveaux. Premièrement, en se plaçant sur le créneau d'artistes " en développement ", Collablive ambitionnait de contribuer d'emblée au renouvellement qualitatif de l'offre au sein de la filière du live en France, en permettant à des publics spécifiques de faire jouer des musiciens de leur choix, notamment dans des zones géographiques situées à l'écart des circuits de tournées habituels (banlieues, villes moyennes). Deuxièmement, à terme, il visait de facto à court-circuiter de nombreux intermédiaires (programmateurs, promoteurs, tourneurs et autres agences de booking, officines médiatiques nationales, locales et spécialisées...) et par conséquent à favoriser l'émergence d'une offre plus diversifiée, plus accessible, et située hors de l'emprise des acteurs industriels oligopolistiques dont on a rappelé plus haut la présence croissante dans cette filière. Troisièmement - et c'était sans doute là le « pari » le plus osé de Collablive - la plateforme devait progressivement contribuer au regroupement des dispositifs existants de subvention publique par la demande, de façon à réimpulser indirectement l'action publique dans cette filière. Ainsi, en lien avec les programmes de développement culturel de collectivités territoriales et d'institutions publiques, et en partenariat avec un réseau établi de salles, cette plateforme devait permettre à des usagers amateurs de musique de participer en ligne à la programmation, au préfinancement et à la promotion de concerts, puis de se retrouver physiquement lors des événements produits. Établie sous la forme d'une association loi 1901, Collablive devait demeurer une structure non-lucrative et pouvait être conçue comme la genèse d'une nouvelle forme de service public. Précisons enfin que le dispositif n'était pas conçu uniquement pour le territoire français ; il était prévu de l'exporter outre-Manche au sein d'un réseau de salles de concert public dès les premières expérimentations.

S'agissant des rôles des différents partenaires du consortium, si les places de la structure de production de spectacles et du développeur informatique et infographique étaient clairement définies, il convient de préciser qu'une certaine ambiguïté a caractérisé les positions des chercheurs du CEMTI ${ }^{10}$. Nous développons davantage ces questions dans la seconde partie, mais précisons d'entrée de jeu que si l'accompagnement d'une plateforme web communautaire en cours de développement offrait des perspectives de recherches empiriques prometteuses (observations des négociations avec les partenaires industriels et institutionnels, accès aux données des usages, entretiens avec les usagers lors 10 Ont pris part à ces travaux Loïc Ballarini, Jacob Matthews, Vincent Rouzé et Jérémy Joseph
Vachet. 
Les recherches critiques appliquées dans le champ des TICN : chimère ou percée ? Éléments de réflexion

des concerts produits ou en dehors, etc.), il impliquait par ailleurs un certain devoir d'orientation déontologique, de façon à assurer que le projet ne soit pas, dès la première occasion, happé par les logiques industrielles évoquées cidessus. De plus, reconnaissons que notre souhait de voir émerger l'instrument de nos recherches nous a conduit, à plusieurs reprises à aller au-delà de la position de simples observateurs ou de "parrains » éthiques.

Nous avons toutefois fixé quatre objectifs clairs pour le projet de recherche, labellisé par la Maison des Sciences de l'Homme Paris Nord en 2012. D'abord, il se proposait de poursuivre l'étude du web collaboratif et des phénomènes du crowdsourcing et du crowdfunding au sein des industries de la culture et de la communication. Deuxièmement, il visait à contribuer aux débats théoriques concernant les "industries créatives ", la structuration et les hypothétiques modélisations des réseaux sociaux qui sous-tendraient ces industries, leurs modes de valorisation et leur position dans le capitalisme contemporain. Troisièmement, il proposait de produire des savoirs empiriques permettant de contribuer aux débats sur la question du financement public des industries culturelles, et plus spécifiquement les filières musicales. Enfin, il visait à concevoir des applications pratiques d'approches critiques existantes des industries de la culture et de la communication, notamment de par le suivi du dispositif Collablive.

Le projet de consortium LLC n'ayant pas été retenu lors de l'appel à projets du Ministère de la Culture "Services numériques culturels innovants 2012 » et n'étant pas parvenu à assurer des sources de financement garantissant la préservation des caractéristiques susmentionnées (pour des raisons que nous développons ci-dessous), ce partenariat a de facto périclité au cours de l'été 2012. Les dix mois de cette "aventure " n'en constituent pas moins une expérience singulière de recherche critique appliquée, dont nous livrons les enseignements dans la partie qui suit.

\section{Des enjeux et antagonismes spécifiques aux recherches critiques appliquées?}

\subsection{Les contraintes du cadre institutionnel}

La constitution de partenariats regroupant industriels et chercheurs se présente désormais comme un critère d'évaluation positive pour un grand nombre d'appels d'offres scientifiques portant sur les TICN. En France, cette tendance est bien sûr liée aux dispositifs mis en place depuis une vingtaine d'années par des gouvernements de droite comme de gauche, liant 
matériellement et idéologiquement la recherche et la performance économique (tels que les fameux « pôles de compétitivité "), mais il faut aussi faire remonter ces choix politiques à la stratégie de Lisbonne au niveau européen. D'après le site officiel de la DGCIS, cette institution a publié plus de 30 appels à projets liés aux TICN depuis 2011, tandis que Cap Digital, le pôle de compétitivité de «la filière des contenus et services numériques ", recense un total de 419 projets labellisés depuis 2006, dont environ $70 \%$ sont des consortia associant des équipes de chercheurs universitaires. Ces chiffres ne sont pas surprenants en soi ; la politique industrielle française promeut depuis de nombreuses années les partenariats entre universités et entreprises privées. Ce qui ne laisse pas d'étonner cependant, c'est la proportion sans cesse croissante de ces projets associant des chercheurs des SHS et la forme précise que prennent ces partenariats.

Avec le développement de ces consortia, il semble en effet qu'on passe de travaux portant principalement sur les usages de TIC déjà commercialisés, menés par des équipes de recherche et «profitant» plus ou moins indirectement aux entreprises, vers des programmes de R\&D associant industriels et chercheurs, et visant la conception de prototypes de biens et services, avec des objectifs de rentabilisation à court terme. En somme, cette transition apparaît comme celle d'une situation où alors que traditionnellement les résultats de recherches sur les TIC étaient épisodiquement introduits au sein des calculs stratégiques des entreprises, la recherche tend dorénavant à devenir précisément ce que produisent des associations ad hoc regroupant des entreprises et des chercheurs.

Or, nous sommes tentés de rapprocher ce constat de celui fait par les auteurs de Capitalisme et industries culturelles il y trente-cinq ans, concernant le phénomène d'externalisation croissante qui caractérise ce secteur, structuré sous la forme d'un oligopole à franges, permettant comme on le sait de transférer une partie du risque lié au caractère incertain de la valorisation des produits culturels vers des structures «indépendantes » (Miège et al., 1978). Certes, l'utilisation par des entreprises de savoirs développés au sein de l'université n'est pas une nouveauté en soi ; on décèle toutefois les indices d'une mutation profonde dans les rapports entre ces deux entités. Privés d'une partie conséquente de leurs financements propres, les laboratoires de recherche en SHS ne seraient-ils pas en passe de se transformer en "viviers " pour un nombre croissant d'entreprises privées qui externalisent une partie de leurs tâches de R\&D vers d'éphémères consortia montées selon leurs intérêts du moment ? Bien qu'il existe encore certains « interstices » (comme en atteste notre propre expérience du consortium $C B$ ), cette mutation touche une variété 
Les recherches critiques appliquées dans le champ des TICN : chimère ou percée ? Éléments de réflexion de disciplines et nous semble particulièrement avancée dans le champ des
recherches portant sur les TICN. ${ }^{11}$

L'organisation du travail au sein de ces consortia mérite par ailleurs d'être brièvement évoquée : on constate en effet de nouvelles formes d'exploitation de la recherche, au travers des processus d'externalisation susmentionnés. Parmi les exemples observés, citons l'emploi de doctorants comme "consultants » (à travers des dispositifs comme les contrats $\mathrm{CIFRE}^{12}$ ), le recours à des doctorants auto-entrepreneurs pour des activités régulières ${ }^{13}$, la force de travail quasiment non rémunérée des étudiants stagiaires. Si cette instrumentalisation des travailleurs moins qualifiés est somme toute peu surprenante, il serait erroné de croire que les chercheurs titulaires soient épargnés. Notons d'abord qu'au sein des consortia, ils fournissent de facto à l'entreprise un "travail gratuit ", comptabilisé parmi leur service de recherche rétribué par l'université. La stagnation de leurs revenus réels et les lourdes charges d'activités annexes (enseignement et gestion administrative) sont sans doute à compter parmi les raisons qui poussent certains à quitter le «vivier » en montant leurs propres

\footnotetext{
11 II nous semble utile de mettre en parallèle ces remarques avec les propositions critiques des géographes Richard Gibson et Natasha Klocker (2004). Ces derniers avancent que les logiques des "industries créatives " s'étendent désormais au champ de l'enseignement supérieur et de la recherche. Les perspectives de "valorisation " des universités dépendraient désormais de leur propension à extraire des revenus de la vente de biens et services symboliques (cours, séminaires, ouvrages, diplômes...), de l'organisation d'événements (conférences et colloques), des droits de propriété intellectuelle et industrielle, sans parler du rôle de leurs marques comme "capital intangible ". De la même façon, les stratégies de "valorisation » individuelle seraient de plus en plus basées sur une logique de star-système, les célébrités académiques monnayant leurs prestations sur un marché mondial tout aussi hautement concurrentiel. (Gibson, Klocker, 2004).

${ }^{12}$ En France, le dispositif CIFRE garantit certes une rémunération correcte et permet à de nombreux thésards de trouver un emploi à l'issue de leurs recherches doctorales. II ne faudrait toutefois pas sous-estimer la charge de travail complémentaire aux recherches doctorales proprement dites, ou la redéfinition des projets de thèse en fonction des demandes de l'organisation, que de tels contrats peuvent impliquer. De plus, des informations recueillies sur la liste de diffusion "profs-sup " montrent que ce dispositif constitue à bien des égards le pic de l'iceberg des formes d'instrumentalisation de doctorants. La mise en place, en France, de "contrats doctoraux", dépendant de chaque établissement, apparaît comme l'occasion d'un renforcement de logiques mandarinales, notamment lorsque le directeur de thèse et le responsable de contrat doctoral se trouvent être la même personne. La relative rareté de ces contrats, en SHS, fait que le contrat " est souvent présenté comme un cadeau qui doit se mériter », comme le signale un responsable d'une association française de doctorants en sciences de gestion et en économie. Si l'on ajoute à cela, enfin, le cas des nombreux doctorants en "CDD recherche ", il est manifeste que les ingrédients sont désormais réunis pour institutionnaliser l'emploi de thésards sur des projets de recherche dont la définition et les enjeux leur échappent pleinement, une situation aux antipodes de l'autonomie que procuraient les allocations de recherche ministérielles et régionales.

${ }^{13}$ C'est ainsi que fut rétribué le travail de recherche de Jérémy Joseph Vachet, cette modalité ayant été recommandée par nos interlocuteurs institutionnels au vu du budget dont nous disposions alors.
} 
start-ups ou en négociant avec les entreprises partenaires de façon à obtenir leur «part du gâteau » (honoraires complémentaires, droits de propriété industrielle ou intellectuelle). Ce phénomène n'est sans doute pas inédit. Dès les années 1990, Robert Boure observait: "plus les occasions de conquérir des positions dans sa discipline sont rares (du fait de l'insuffisance des postes, du petit nombre de lieux "légitimes": équipes, systèmes de publication, colloques, prix scientifiques...), plus la tentation est grande de rechercher des compensations dans des champs non scientifiques » (Boure, 1997, p. 247). On peut émettre l'hypothèse que le contexte idéologique et socio-économique actuel encourage d'autant plus cette recherche de compensations, qui va de pair avec le développement d'un certain entrepreneuriat académique.

Au cours des recherches menées dans le cadre du consortium $L L C$, ces questions se sont posées à plusieurs reprises, du fait de la précarité et/ou des ambiguïtés de positionnement des chercheurs (et du responsable de Collablive, lui-même ancien étudiant de l'Université Paris 8). En dernière instance, nos choix déontologiques et le projet d'une recherche critique pleinement assumée auront eu raison de ces tentations de transformer la plateforme en entreprise privée, mais les logiques propres à ces partenariats - et au soutien public qui leur est accordé - abondent très clairement en ce sens.

On ne saurait en effet sous-estimer le rôle joué par l'État en faveur des collusions entre sphères économique et académique qui caractérisent ces consortia. Dans le cadre du programme de recherches LLC, nous avons constaté à quel point les acteurs publics sollicités et rencontrés par le porteur de projet Collablive s'efforçaient d'ajuster celui-ci aux desiderata des logiques socio-économiques dominantes. A titre d'exemple, la principale cause de notre élimination lors de l'appel à projets "Services numériques culturels innovants 2012 " s'avéra être la forme juridique de la structure sur laquelle s'appuyait la plateforme, une association loi 1901. D'après nos interlocuteurs, celle-ci laissait planer des soupçons de "manque de sérieux » que seule la prise de risque financière liée à une création d'entreprise pouvait écarter. De la même façon, nos observations de discussions avec des responsables de Cap Digital ont révélé leurs fortes incitations à transformer le projet en un service B2B (business to business) de captation de données sur les usages effectifs et potentiels de salles de concerts. D'après les discours des représentants de cette institution parapublique, le financement du consortium -qu'il provienne d'acteurs institutionnels privés ou publics - ne pouvait être garanti que si le prototype offrait non seulement des preuves de viabilité économique, mais surtout d'une rentabilité à court terme. Quand bien même les divers interlocuteurs publics (Ministère de la Culture, CNV, Scientipole Initiative, Oséo, représentants de collectivités territoriales...) percevaient clairement les apports 
Les recherches critiques appliquées dans le champ des TICN : chimère ou percée ? Éléments de réflexion

du projet en termes de diversification de l'offre culturelle et son intérêt expérimental, leur «logiciel » n'admettait aucunement qu'il puisse se déployer en dehors des sentiers battus de la marchandisation.

Ces observations révèlent la prégnance croissante du paradigme des «industries créatives ", pour lequel le financement et le soutien publics aux projets culturels dépend avant tout autre paramètre, de leurs capacités (supposées ou réelles) à générer de la valeur et à " soutenir la croissance » (Bouquillion, 2012). De façon plus générale, nous proposons qu'elles renvoient vers une nouvelle forme de rationalité de l'action publique, basée sur la croyance en la supériorité managériale du privé sur le public, que la sociologue Béatrice Hibou appelle le principe d'homothétie public-privé (Hibou, 2012). La recherche critique dans le domaine des TICN se doit d'appréhender cette conception, notamment du point de vue de ses déclinaisons au niveau de la relation industrie / recherche. Ainsi celle-ci s'appuierait sur des intérêts réciproques et communs : l'industrie apporte son "terrain » aux chercheurs (comme "matière première " pour la valorisation de ce qui est conçu comme une «industrie universitaire "), en échange de quoi la recherche bénéficie immédiatement à l'industrie (en lui permettant un abaissement des coûts, en vue d'une valorisation optimale de sa production).

En affirmant de façon simplificatrice que la création de valeur profiterait à tous - entreprises privées, laboratoires de recherche, chercheurs et usagers des TICN - une telle vision s'efforce de masquer les contradictions inhérentes au capitalisme contemporain. Elle paraît, en ce sens, tout à fait antinomique avec les principes et exigences de la recherche critique ${ }^{14}$. Elle se présente pourtant comme l'une des principales justifications des politiques publiques, aussi bien en direction de l'enseignement supérieur et la recherche que des « industries créatives ». Si des chercheurs, tout comme des travailleurs créatifs et porteurs de projets culturels, souhaitent bénéficier de financements publics, ils sont désormais sommés de fournir des indices mesurables de leur efficacité (notamment en matière de création d'emplois), des preuves de leur contribution à l'effort compétitif régional, national ou européen (selon les cas).

\footnotetext{
14 La principale auto-critique que nous formulons suite à l'expérience des recherches menées au sein du programme $C B$ est que nous avons de facto collaboré à la réorientation des politiques publiques qui permet à une société comme $C C$ de bénéficier d'une partie, si infime soit-elle, de la richesse nationale socialisée pour développer un service privé et marchand. Même si cette "association" nous a permis de développer des analyses critiques du crowdfunding, on peut légitimement se demander a posteriori si «le jeu en valait la chandelle».
} 


\subsection{Les ambiguïtés de la position du chercheur critique dans le champ des TICN}

L'approche critique exige à la fois une distanciation vis-à-vis de l'objet étudié et une réflexivité particulière, quant à la position du chercheur (Bourdieu, 1984, 1997 ; Bourdieu, Wacquant, 1992 ; Corcuff, 2003). Les réflexions que nous livrons ci-dessous, à partir de nos expériences singulières de recherche critique, peuvent être mises en parallèle avec les considérations de Michel Foucault, pour qui la légitimité du chercheur critique repose sur au moins trois conditions: l'inscription au sein de l'institution universitaire, le questionnement de soi et du sens de ses actions, mais également la possibilité de disposer de fonds suffisants pour mener à bien des recherches désintéressées (Foucault, 1981).

Un constat s'impose : nos expériences de recherche critique dans le champ des TICN se sont appuyés précisément sur des cadres institutionnels qui ne permettent pas d'être financés autrement que par des modalités intrinsèquement problématiques, eu égard les deux premières conditions énoncées. Ces expériences ont par conséquent comporté une nécessaire " gestion " de tensions particulières et exigé une vigilance accrue, s'agissant de la position du chercheur.

En effet, l'activité de recherche dans le cadre de consortia implique, en principe, de travailler directement aux côtés des acteurs industriels, de partager à la fois les terrains (par exemple, les données des utilisateurs) et les résultats. Le chercheur y est donc considéré comme l'un des moteurs de l'innovation industrielle. Comme nous l'avons signalé, notre propre expérience au sein du consortium $C B$ a certes présenté un caractère atypique à cet égard, dans la mesure où nous n'étions pas associés à la conception du prototype de service visé par ce programme. Toutefois, plusieurs observations méritent d'être soulignées à ce niveau, s'agissant des attitudes et des discours sur la recherche formulés par les entrepreneurs et représentants des institutions étatiques.

Premièrement, nous avons constaté que la figure du chercheur - en tant que garant scientifique, voire éthique, de la production de savoirs et de techniques était fréquemment mobilisée. Ce fut par exemple le cas lors des réunions avec les responsables de CrowdCulture et l'interlocuteur de la DGCIS; notre présence était requise, alors même que le caractère tout à fait facultatif de nos contributions et propositions était devenu manifeste au fil des mois. S'était ainsi établi un étrange jeu de représentations dont personne ne pouvait réellement être dupe : entrepreneurs, fonctionnaire et chercheurs simulions tous trois, dans une large mesure, ce à quoi pouvait ressembler une réunion de consortium "régulière ". De la même façon, nous avons été sollicités en urgence lorsque le secrétariat d'État à l'économie numérique décida d'organiser une conférence de 
Les recherches critiques appliquées dans le champ des TICN : chimère ou percée ? Éléments de réflexion

presse pour présenter, à mi-parcours, les projets soutenus dans le cadre du "volet numérique » du plan de relance national. Pour la DGCIS comme pour notre partenaire, la présence de chercheurs était d'autant plus indispensable que cet exercice de public relations était conduit par la secrétaire d'État en personne. Notre expérience tend à montrer que si l'intégration de chercheurs au sein de consortia se fait sur la base des besoins concrets des entreprises (pour la mise en œuvre de leurs propres stratégies de conception et de production ${ }^{15}$ ), elle passe également par la mise en scène de représentations symboliques permettant de consolider les relations entre acteurs industriels et institutions publiques (dans ce cas la DGCIS) - dont dépend le financement d'une partie de l'activité de l'entreprise.

S'agissant de nouvelles formes d'exercice de la recherche rencontrées, une analogie intéressante à été proposée par Luc Boltanski et Ève Chiapello :

"Le chercheur se retrouve dans cette situation unique, celle du néo-manager (...). Le chercheur n'est-il pas lui aussi, aujourd'hui, un homme de réseaux à la recherche de producteurs, dont les projets réclament, pour s'accomplir, la mise en place de montages coûteux, hétérogènes et complexes, la capacité à s'entendre avec des acteurs distants et multiples, occupant des positions très diverses (de l'élu local, au chef d'entreprise, en passant par l'attaché de ministère - qu'il doit intéresser, convaincre, séduire " (Boltanski, Chiapello, 1999, p. 438).

Nos observations rendent clairement compte de ces logiques de fonctionnement par projet (polyvalence, auto-contrôle, développement de l'autonomie et de la flexibilité, etc.). Dans le cadre du consortium LLC, nous nous sommes ainsi trouvés dans la position de devoir convaincre de nouveaux

\footnotetext{
15 Dans notre cas, c'est curieusement par la négative que cette première forme d'intégration s'est "donnée à voir ". Ainsi, la proposition faite oralement, en présence du représentant de la DGCIS, d'attribuer un espace de travail au doctorant Efstathios Sinos au sein de l'entreprise, ne s'est jamais concrétisée, soi-disant par "manque de place " dans leurs locaux. Lorsque nous avons souhaité avoir accès aux données d'usage de la version béta de la plateforme CrowdBooks, nos partenaires ont tergiversé durant plusieurs semaines, avant de nous accorder finalement l'accès à des données déjà anciennes (et partiellement censurées) provenant de leur plateforme de crowdfunding de musique. De même, quand nous avons émis la proposition de mener des entretiens avec des représentants de la maison d'édition associée à l'opération CrowdBooks, nos partenaires "proposèrent" une solution consistant à effectuer un premier filtrage des points que nous souhaitions aborder, se disant capables de répondre eux-mêmes à une partie de nos questions et justifiant cette nouvelle censure par le fait que les dirigeants de cette société étaient fort occupés et ne disposaient sans doute pas du temps nécessaire pour nous répondre...
} 
Jacob T. MATTHEWS et Jérémy Joseph VACHET

acteurs financeurs de l'utilité de nos recherches, ce qui, dans certains cas, revenait de facto à arguer de la rentabilité économique à court terme de la plateforme Collablive. II en fut de même lorsque, pour permettre la mise en place d'expérimentations de cette plateforme outre-Manche, nous développions un discours proche de celui d'un représentant commercial, slides de l'interface web à l'appui, de manière à "appâter " les gestionnaires d'une salle potentiellement partenaire. II s'agit là de formes de responsabilisation du chercheur quant au succès ou à l'échec de ces projets de recherches, qui dépendent in fine de critères purement économiques. Ces modalités d'exercice de la recherche impliquent, de concert avec l'acquisition de compétences supplémentaires, l'assimilation de nouvelles formes de rationalité : le choix même des objets de recherche peut varier en fonction des possibilités de financement, car derrière cette logique se cache surtout le spectre de ne pas pouvoir mener à terme les recherches. En effet, dans la perspective de consortia relativement éphémères (nos observations portent sur des projets d'une durée moyenne de quinze mois), les possibilités de conduire à bien des recherches sont clairement remises en question, avec pour corollaire le sentiment épuisant de courir de projet en projet.

Un autre indicateur de cette forme d'organisation du travail par projet, et de l'incursion des normes managériales, est l'augmentation significative des tâches bureaucratiques (rédaction de dossiers et rapports, gestion des recrutements et vérification des paiements, organisation pratique des missions de recherche, etc.) en vue de consolider des travaux en cours ou d'élaborer de nouvelles demandes de financement. Ces logiques bureaucratiques ne constituent pas en soi une nouveauté (la recherche "administrative " s'efforce depuis longtemps d'assimiler l'activité scientifique à la sphère gestionnaire). Elles semblent cependant amplifiées du fait de la généralisation de pratiques managériales issues du secteur privé (Hibou, 2012). Chaque nouveau projet nécessite une somme propre de tâches répétitives (montage de dossier dans le strict respect des normes des appels), gangrenant une part non négligeable du temps de recherche. D'autre part, la faible durée de ces projets et l'incertitude quant à leur réalisation peuvent évidemment contribuer à la distorsion des thématiques et objets de recherches, dans le seul but de répondre aux critères d'évaluation supposés ou réels.

Instrumentalisation symbolique et/ou matérielle, autonomisation et responsabilisation logistique accrues, acquisition ou improvisation de compétences promotionnelles et gestionnaires... Sans vouloir aucunement "dramatiser » ces divers points, ni en tirer des généralisations hâtives, il nous semble important d'en faire part ici, de façon à nourrir la discussion s'agissant des conditions de production des recherches critiques. Partant du principe que 
Les recherches critiques appliquées dans le champ des TICN : chimère ou percée ? Éléments de réflexion

nous ne serions pas les seuls à en faire l'expérience, il nous paraît important de s'interroger sur les éventuelles conséquences que peuvent avoir ces phénomènes du point de vue de nos exigences de distanciation et de réflexivité critiques.

\section{Conclusion: vers une dialectique de la participation et de la distanciation propre aux recherches critiques appliquées ?}

Les discussions sur l'équilibre périlleux entre distance et engagement ne sont pas neuves et notre propos n'est pas de définir ici une quelconque conduite à tenir, pas plus que nous ne pourrions, en quelques lignes, prétendre résoudre la question épineuse du rapport entre théorie et praxis critiques. Les expériences de recherches critiques appliquées évoquées dans cet article nous permettent néanmoins de tirer quelques conclusions partielles, que nous espérons utiles à d'autres chercheurs hétérodoxes confrontés à ces mêmes enjeux et antagonismes.

La première question à laquelle nous faisons face suite à ces travaux est celle de la pertinence de notre participation au processus institutionnel qui conditionne en grande partie la faisabilité matérielle de recherches critiques. Comment et pourquoi prendre part à ce qui paraît relever, au mieux, d'une gigantesque farce, au pire, d'une entreprise de liquidation systématique de la posture critique ? Les appels à projets scientifiques les plus courants dans le domaine des TICN soumettent d'emblée les candidats à des contraintes formelles, stylistiques, dont il conviendrait d'analyser le caractère performatif et les implications idéologiques. Dans les deux cas évoqués, nous avons fait l'expérience, à ce niveau précis, d'une violence symbolique certaine, soit lorsqu'il s'agissait de "parler la langue barbare de la R\&D», soit lorsqu'on tentait de faire entrer le projet atypique $L L C$ au sein des cases rigides et hostiles de l'appel à projets susmentionné, de lui attribuer ce faisant l'apparence réglementaire du consortium. Sans même nous préoccuper ici du bien fondé des thèmes de ces appels à projets, soulignons que les chercheurs, impliqués par ailleurs dans des réseaux socio-professionnels contraignants, sont de facto tenus d'anticiper ce que les instances d'évaluation attendent d'eux. Cette question touche au cœur même de la notion d'indépendance intellectuelle; elle doit être prise en compte très sérieusement. Deux options génériques semblent s'offrir aux chercheurs critiques: accepter de participer en s'efforçant de subvertir ces contraintes dans la pratique de recherche et dans la production 
ultérieure (comme nous avons tâché de le faire ${ }^{16}$ ) ou refuser toute collaboration avec ces logiques, avec dès lors comme point de fuite le terrain de la lutte politique et syndicale.

Notre seconde conclusion a trait plus spécifiquement aux études portant sur les TICN. Du fait de la volatilité de ce champ (acteurs industriels mouvants, phénomènes socio-économiques émergents, innovations socio-techniques), on peut se demander si les recherches critiques n'ont pas intérêt à jouer en quelque sorte "double jeu ", afin d'être au plus près des processus observés. Comment en effet rendre compte des évolutions sans prendre part aux consortia où celles-ci se déploient? Ces tactiques, que nous avons pleinement assumées et qui relèvent au fond de formes d'" espionnage", ne sont aucunement inédites dans les SHS, mais on peut penser qu'elles seront amenées à se multiplier dans les années à venir. Nos expériences montrent qu'elles exigent des savoir-faire particuliers (qui requièrent encore un effort considérable de compilation et de systématisation de la part des chercheurs engagés dans cette voie) ; face à la méfiance endémique de "partenaires » industriels évoluant dans des contextes hautement concurrentiels, les résultats d'observations effectuées lors de conférences professionnelles ou de séminaires privés sont souvent bien en-deçà des espérances initiales. Comme nous l'avons en outre illustré, ces tactiques de recherche critique appliquée présentent le risque d'une certaine «schizophrénie", pour les jeunes chercheurs comme pour les plus expérimentés.

Nous souhaitons enfin réitérer notre accord avec la proposition d'Olivier Voirol, s'agissant de la nécessité de préserver, au centre de ces modalités de recherche, des repères éthiques "permettant, d'une part, de concevoir des formes émancipatrices de créations culturelles et d'autre part, de mener une critique systématique de ce qui contribue à les oblitérer, les empêcher ou les détruire » (Voirol, 2010, p. 32). Nos travaux d'accompagnement de la structure Collablive ont été marqués par des discussions nombreuses et vives autour des arbitrages stratégiques propres au développement et au lancement de la plateforme (choix techniques, artistiques et géographiques, choix des partenaires et prestataires, question de la captation de données, etc.). Ces échanges collectifs quasi incessants ont été souvent marqués par une confusion dérangeante des rôles. Ils constituaient cependant et par-dessus tout l'occasion de forger et de consolider, à notre niveau, ce "référent pratique " dont parle Voirol et sans lequel la critique se trouve coupée de sa visée émancipatrice,

\footnotetext{
${ }^{16}$ En l'occurrence, le lecteur pourra juger de la pertinence de cette démarche à partir des résultats et propositions formulés dans notre ouvrage collectif La culture par les foules: le crowdfunding et le crowdsourcing en question (Matthews, Rouzé, Vachet, 2014).
} 
Les recherches critiques appliquées dans le champ des TICN : chimère ou percée ? Éléments de réflexion

impuissante face aux logiques du capitalisme. Que ce projet n'ait pas tenu toutes ses promesses, c'est un fait ; il a toutefois eu le profond mérite de nous rappeler que la pensée critique n'est en aucun cas figée et qu'elle puise sa force de confrontations singulières.

\section{Références bibliographiques}

ADORNO, T., 1951, Minima Moralia, Francfort, Suhrkamp Verlag.

ADORNO, T., 1993, “Messages in a bottle », New Left Review, n200, pp. $5 \square 14$.

BOLTANSKI, L., CHIAPELLO, E., 1999, Le nouvel esprit du capitalisme, Paris, Gallimard.

BOUQUILLION P. (dir.), 2012, Creative Industries, Creative Economy, des notions à traduire, Vincennes, Presses Universitaires de Vincennes.

BOURDIEU P., 1984, Questions de sociologie, Paris, Éditions de Minuit.

BOURDIEU P., 1997, Méditations pascaliennes, Paris, Le Seuil.

BOURDIEU P. et L. WACQUANT, 1992, Réponses. Pour une anthropologie réflexive, Paris, Le Seuil.

BOURE R.,1997, "Les sciences de l'information et de la communication au risque de l'expertise ? Sur et sous des pratiques scientifiques ", Réseaux, vol. $15 n^{\circ} 82-83$, pp. 233-254.

BRENNAN M. et E. WEBSTER, 2011, "Why concert promoters matter ", Scottish Music Review, vol. 2, n9, pp. 1-25.

CORCUFF P., 2003, Bourdieu autrement. Fragilités d'un sociologue de combat, Paris, Textuel.

DUFY G., 2012, "L'économie du secteur du concert en France à l'heure du numérique " dans MATTHEWS J. et L. PERTICOZ, 2012, L'industrie musicale à l'aube du $21^{e}$ siècle, Paris, L'Harmattan.

FLICHY P., 2010, Le sacre de l'amateur : sociologie des passions ordinaires à l'ère numérique, Paris, Le Seuil.

FOUCAULT M., 1981, Subjectivité et vérité, Paris, Annuaire du collège de France, 81e année, Histoire des systèmes de pensée.

FRITH S., 2007, Taking popular music seriously, Farnham (Surrey), Ashgate Publishing. 
GIBSON C. et N. KLOCKER, 2004, "Academic publishing as "creative" industry, and recent discourses of "creative economies": some critical reflections ", Area, vol. 36, n 4 , pp. 423-434.

GUIBERT G., 2006, La Production de la culture. Le cas des musiques amplifiées en France. Genèses, structurations, industries, alternatives. Volume ! La revue des musiques populaires.

HIBOU B., 2012, La bureaucratisation du monde à l'ère néo-libérale, Paris, La Découverte.

JENEMANN D., 2007, Adorno in America, Minneapolis, Minneapolis, University of Minnesota Press.

JENKINS H., 2006, Convergence Culture: Where Old and New Media Collide, New York, New York University Press.

MATTHEWS J., 2009, "You can't blame the youth : pistes de réflexion autour de processus de starisation dans l'industrie musicale ", dans M. MATHIEN (dir.), Les jeunes dans les médias en Europe, Bruxelles, Bruylant, pp. 199213.

MATTHEWS J., V. ROUZE et J. VACHET, 2014, La culture par les foules : le crowdfunding et le crowdsourcing en question, Paris, MKF Editions.

MIEGE B., A. HUET, J. ION, A. LEFEBVRE et R. PERRON, 1978, Capitalisme et industries culturelles, Grenoble, Presses Universitaires de Grenoble.

VOIROL O., 2010, "La Théorie critique des médias de l'École de Francfort: une relecture », Mouvements, n61, pp. 23-32. 